\title{
Fluxograma para o manejo de pessoas idosas com alterações de deglutição pós- acidente vascular cerebral
}

\author{
Fluxogram for the management of the elderly with altered deglutition after cerebrovascular \\ accident
}

Diagrama de flujo para el manejo de personas mayores con cambios de ingestión tras accidente vascular cerebral Universidade Federal da Paraíba, Brasil E-mail: nyellisonobrega @ hotmail.com

Ronaldo Bezerra de Queiroz

ORCID: https://orcid.org/0000-0001-7983-9666 Universidade Federal da Paraíba, Brasil

E-mail: queirozrbq@gmail.com

Adriana Queiroga Sarmento

ORCID: https://orcid.org/0000-0002-8714-7923 Universidade Federal da Paraíba, Brasil E-mail: aqsguerra@gmail.com

Olívia Galvão Lucena Ferreira

ORCID: https://orcid.org/0000-0002-8490-2444 Universidade Federal da Paraíba, Brasil

E-mail: oliviaglf@hotmail.com

\section{Resumo}

Objetivo: Construir um fluxograma para o manejo de pessoas idosas com alterações de deglutição pós acidente vascular cerebral. Métodos: Estudo metodológico realizado em três etapas: realização de revisão integrativa da literatura; avaliação de 38 prontuários acerca de variáveis sociodemográficas, clínicas e prevalência de alterações de deglutição de idosos pós acidente vascular cerebral; e construção do fluxograma para o manejo de idosos com alterações da deglutição pós acidente vascular cerebral, com base nas duas primeiras etapas. Resultados: O fluxo inicia com o acolhimento da pessoa idosa pós acidente vascular cerebral na Atenção Primária à Saúde, onde será avaliada por profissional de saúde. Ao verificar alterações na deglutição, o idoso será encaminhado para tratamento com fisioterapeuta e fonoaudiólogo ou nutricionista, psicólogo e fonoaudiólogo, conforme alterações pulmonares ou nutricionais e alimentares, respectivamente. Conclusão: O fluxograma auxilia os profissionais de saúde no encaminhamento dos idosos com essa condição, propiciando tratamento adequado pela equipe multidisciplinar.

Palavras-chave:Deglutição; Transtornos de deglutição; Acidente vascular cerebral; Idoso; Equipe de assistência ao paciente.

\footnotetext{
Abstract

Objective: To build a flowchart for the management of elderly people with swallowing disorders after stroke. Methods: Methodological study carried out in three stages: carrying out an integrative literature review; evaluation of 38 medical records about sociodemographic and clinical variables and the prevalence of swallowing disorders in elderly people after a stroke; and construction of a flowchart for the management of elderly people with swallowing
} 
disorders after a stroke, based on the first two steps. Results: The flow starts with the reception of the elderly person after a stroke in Primary Health Care, where they will be evaluated by a health professional. When checking changes in swallowing, the elderly will be referred for treatment with a physiotherapist and speech therapist or nutritionist, psychologist and speech therapist, according to pulmonary or nutritional and dietary changes, respectively. Conclusion: The flowchart helps health professionals in referring the elderly with this condition, providing adequate treatment by the multidisciplinary team.

Keywords: Deglutition; Swallowing disorders; Stroke; Elderly; Patient care team.

\begin{abstract}
Resumen
Objective: Elaborar un diagrama de flujo para el manejo de personas mayores con trastornos de la deglución después de un accidente cerebrovascular. Métodos: Estudio metodológico realizado en tres etapas: realización de una revisión integradora de la literatura; evaluación de 38 historias clínicas sobre variables sociodemográficas, clínicas y prevalencia de trastornos de la deglución en ancianos tras un ictus; y construcción de un diagrama de flujo para el manejo de personas mayores con trastornos de la deglución después de un ictus, basado en los dos primeros pasos. Resultados: El flujo se inicia con la recepción del anciano tras un ictus en Atención Primaria de Salud, donde será evaluado por un profesional de la salud. Al comprobar los cambios en la deglución, los ancianos serán remitidos para tratamiento con fisioterapeuta y logopeda o nutricionista, psicólogo y logopeda, según cambios pulmonares o nutricionales y dietéticos, respectivamente. Conclusión: El diagrama de flujo ayuda a los profesionales de la salud a derivar a los ancianos con esta condición, brindando un tratamiento adecuado por parte del equipo multidisciplinario.

Palabras clave: Deglutição; Transtornos de deglutição; Acidente vascular cerebral; Idoso; Equipe de assistência ao paciente.
\end{abstract}

\title{
1. Introdução
}

A senescência ou senectude está relacionada às alterações orgânicas, funcionais e psicológicas próprias do envelhecimento normal. E a senilidade é caracterizada por modificações determinadas por afecções que frequentemente acometem a pessoa idosa, e é, por vezes, extremamente difícil (Oms, 2015).

O processo de envelhecimento e sua consequência natural, a velhice, envolvem o declínio das funções do corpo. O envelhecimento gera a necessidade de um olhar mais dinâmico sobre os idosos e suas consequências naturais, como também sobre as morbidades mais frequentes, principalmente quando o indivíduo não possui uma boa qualidade de vida e o devido cuidado com a sua saúde. Uma patologia cada vez mais frequente é o Acidente vascular cerebral (AVC), que se encontra entre os fatores de risco da velhice e representa a primeira causa de morte e incapacidade no país, e gera grande impacto econômico e social (Paixão \& Sarquis, 2018); (Mourão \& Lemos, 2016).

O AVC é uma doença cerebrovascular, que representa uma das principais causas de óbitos entre as pessoas com mais de 60 anos e a segunda entre os 15-59 anos. No Brasil, apesar da redução nas taxas de mortalidade, ainda é a principal causa de morte (Ribeiro, 2018). O AVC é a terceira causa mais comum de óbito pelas estatísticas divulgadas pela American Heart Association, e é apenas superada pelas doenças cardíacas e cânceres ${ }^{5}$. Uma das sequelas do AVC em idosos é a disfagia, que é uma alteração que pode comprometer todo o processo da deglutição, no que concerne às fases e aos eventos desta função, e pode acometer até 1/3 da população, em graus de severidade variáveis (Winstein \& Crammer, 2019).

As alterações do processo da alimentação merecem atenção especial, uma vez que estão diretamente relacionadas com a nutrição e a qualidade de vida, no idoso. É notória a incidência de transtornos de deglutição como sequela de Acidente vascular cerebral (AVC). O processo de deglutição caracteriza-se por sucessivos movimentos envolvendo a atuação de diversos circuitos neurais, e para que esta realização sincrônica ocorra, desde sinapses entre o córtex cerebral, o cerebelo, e demais estruturas que são essenciais para que este processo seja desencadeado de forma eficaz e segura (Cunha \& Chaves, 2018).

As alterações de deglutição ou disfagias podem classificar-se em: orofaríngea ou alta, quando existem alterações e mudanças na fase oral ou faríngea da deglutição, sendo comum em casos de AVC, ou baixa ou esofagiana, quando existem alterações na fase esofágica da deglutição. A Disfagia orofaríngea pode ser identificada ainda de acordo com a etiologia em: 
neurogênica, causada por doenças neurológicas ou traumas; mecânica, que ocorre quando há perda do controle do bolo pelas estruturas necessárias para completar uma deglutição normal; e psicogênica, por causas emocionais e psíquicas (Silva, 2006); (Sousa \& Ferraz, 2018).

É importante ressaltar a importância do direcionamento ao profissional de fonoaudiologia, após diagnóstico imediato. Os procedimentos realizados pelo profissional de fonoaudiologia nos casos de alterações de deglutição no pós-AVC incluem técnicas não invasivas de observação clínica, avaliação das estruturas orais e alimentação com avaliação funcional, e ausculta cervical. Dependendo dos resultados da avaliação, monitoramento contínuo da deglutição e reavaliação pode ser necessário, além de exames, caso necessite, como a videofluroscopia da deglutição (Winstein \&, Cramer, 2018).

Assim sendo, surgiu o interesse em construir um fluxograma para o manejo de pessoas idosas com alterações de deglutição que sofreram Acidente vascular cerebral a fim de contribuir para o melhor direcionamento do idoso no pós-AVC, ao profissional de fonoaudiologia e equipe multidisciplinar, para que seja realizada a assistência integral, prevenindo agravos e complicações, minimizando as sequelas e viabilizando o processo de recuperação mais rápido.

\section{Metodologia}

Trata-se de uma pesquisa documental de abordagem qualitativa (Ludke \& Andre, 2013); (Estrela, 2018) realizada a partir da análise de prontuários de um serviço de saúde, visando a conhecer a prevalência das alterações de deglutição pós AVC em idosos assistidos em um serviço de atendimento domiciliar que subsidiou a elaboração de um fluxograma para o manejo de pessoas idosas com alterações de deglutição pós-AVC.

A pesquisa documental foi realizada em um serviço de saúde que compõe a média complexidade (Serviço de Atenção Domiciliar/SAD), localizado na cidade de João Pessoa-PB. O serviço foi devidamente escolhido pelo fato de ser a porta de entrada dos usuários idosos que necessitam de assistência, ao serviço de saúde, quando acometidos por AVC. Vale salientar que esse serviço faz parte do Sistema Único de Saúde (SUS), destituído de caráter privado, prestando assistência à comunidade nos níveis primário e secundário. Esse serviço possui um elevado perfil epidemiológico de idosos acometidos por AVC e apresentando transtornos de deglutição.

Como critérios de inclusão para participação no estudo, foram estabelecidos: idosos de ambos os sexos, com idade superior a 60 anos, acometidos por AVC, que apresentassem registros de alterações de deglutição, assistidos pelo serviço de atenção domiciliar no ano de 2017. Como critério de exclusão para participação na pesquisa: prontuários com preenchimento incompleto dos dados. Desta forma, a amostra foi composta por 38 prontuários, doas quais se analisaram dados sociodemográficos (sexo, idade, distrito sanitário que pertence; tipo de AVC (patologia primária e secundária); informações acerca do cuidador; com quem reside a pessoa idosa; prevalência de alterações de deglutição; principais queixas nos aspectos respiratórios, nutricionais e alimentares, de deglutição; referências e contrarreferências para os serviços de saúde; e a indicação para o profissional de fonoaudiologia.

O presente estudo foi submetido à avaliação do Comitê de Ética do Centro de Ciências da Saúde (CCS) e aprovado sob n ${ }^{\circ} 2.190 .153$ de 27 de julho, CAAE; 67103917.6.0000.5188. A presente pesquisa foi norteada pelas Diretrizes e Normas Regulamentadoras dispostas na Resolução 466/2012 do Conselho Nacional de Saúde, que trata de pesquisas envolvendo seres humanos no cenário brasileiro.

Salienta-se que, segundo a Resolução 466/12, toda pesquisa que envolve seres humanos poderá oferecer riscos. Não obstante, os riscos potenciais desta pesquisa não causaram danos de grande magnitude aos participantes, sendo necessário o anonimato aos participantes, tendo em vista a coleta ser realizada em prontuários de um serviço de saúde. Os possíveis eventos danosos que os participantes poderiam manifestar foram constrangimento ou desconforto; no entanto, caso o participante se sentisse constrangido ou desistisse de sua participação, a conduta adotada seria interrupção da pesquisa pelo pesquisador, sem 
acarretar prejuízo ao participante e à pesquisa; e este poderia ser encaminhado, caso necessário, e se assim o participante desejasse, para uma assistência especializada, sem nenhum ônus.

\section{Resultados}

Foram analisados 38 prontuários de pessoas idosas assistidas pelo serviço de atenção domiciliar do município de João Pessoa no período do ano de 2017, onde foi possível observar a prevalência do sexo masculino para o acometimento do AVC (57,9\%), na faixa etária entre 71 a 80 anos $(36,8 \%)$.

Diversas pesquisas referem o sexo masculino como predominante e ainda na faixa etária de 70 a 85 anos de idade com um percentual de 60\% no desenvolvimento do AVC. Na literatura brasileira, há vários estudos que afirmam ser o sexo masculino o predominante entre os indivíduos acometidos por AVC. Pode-se citar a dificuldade de os idosos do sexo masculino optarem em ir realizar consultas rotineiras nos estabelecimentos da rede de atenção à saúde, ou estarem envolvidos em grupo de idosos que visam a realizar estratégias de promoção e prevenção à saúde, e, assim, modificarem seus hábitos de vida (Cunha \& Chaves, 2018).

Quanto à patologia primária, 26,3\% tiveram o AVC do tipo isquêmico e 10,5\% o AVC do tipo hemorrágico, no entanto, $63,2 \%$ não tinha registro do tipo de AVC. No que se refere a patologia secundária, $71,1 \%$ tinham hipertensão arterial e $23,7 \%$ lesão por pressão.

No concernente ao predomínio de AVC isquêmico, essa situação coincide com outros estudos encontrados que contemplam uma amostra de $65 \%$. Visto que o AVCI apresenta sequelas que podem ser passageiras ou transitórias, tendo maior viabilidade no diagnóstico clínico, salienta-se a necessidade de realizar os exames complementares para auxiliar o diagnóstico (Chaves \& Albuquerque, 2020); (Schettino \& Friche, 2020) Observa-se que muitos prontuários analisados não especificaram o tipo de AVC. Esse fato pode ser explicado pelas condições precárias relacionadas aos recursos de saúde, que associam o diagnóstico diferencial aos exames de imagem como tomografia computadorizada de crânio, o qual não está presente em todos os serviços de atenção à saúde que recebem esta demanda de idosos acometidos por AVC. Outro fator que pode contribuir com a ausência desse dado é a dificuldade do atendimento especializado, por neurologista, para estes pacientes, fato relacionado à pequena mão de obra especializada, uma vez que a demanda de médicos neurologistas é restrita para a necessidade dos usuários atendidos pelo SUS (Chaves \& Albuquerque, 2020).

Quanto à observação com quem residiam esses idosos, foi observado que a maioria $(68,4 \%)$ residia com filhos, mesmo aqueles que possuem cônjuges. Sendo a figura do cuidador 65,8\% filho (a), 28,9\% cônjuge e 5,3\% irmão.

Quando investigado o encaminhamento para o profissional de fonoaudiologia, 57,9\% dos idosos foram encaminhados pelos enfermeiros do SAD, seguidos de $21 \%$ pela equipe da ESF, 10,5\% pelos médicos do SAD.

Ressalta-se a importância do encaminhamento da pessoa idosa com alterações na deglutição pós-AVC ao profissional de fonoaudiologia para a realização de anamnese e avaliação clínica, e, consequentemente, proporcionar reabilitação, treinamento, adaptação, compensação, treinamento, gerenciamento e ainda a orientação acerca da dieta alimentar, e de seu manejo durante a alimentação. Um processo de intervenção fonoaudiológica pode provocar mudanças significativas na saúde e, assim, proporcionar qualidade de vida alimentar à pessoa idos (Silva, 2006).

Quanto aos aspectos relacionados às alterações de deglutição pós-AVC extraídas dos prontuários, foi possível observar aspectos pulmonares, nutricionais, alimentares e de deglutição. Quanto aos aspectos pulmonares, 84,4\% foram classificados como estáveis, seguidos por somente hipersecretivo e hipersecretivo, associado à broncoaspiração, suporte ventilatório e traqueostomia, sendo $2,6 \%$ cada classificação.

A maior incidência de idosos classificados como estáveis pode ser explicada pelo fato de que, para ser inserido na assistência domiciliar, o idoso necessita ter estabilidade pulmonar e/ou suporte necessário para receber adequado cuidado às 
suas condições pulmonares, a exemplo de recursos como: aspirador traqueal, oxigênio, cama hospitalar, entre outros (Magalhães \& Bilton, 2014).

Quanto aos aspectos nutricionais, 86,9\% foram classificados como estado regular, seguidos por 7,9\% caquexia, 2,6\% risco de desnutrição e 2,6\% de sobrepeso.

Magalhães e Bilton (2014) relatam que 50\% dos pacientes idosos pós-AVC apresentam alterações de deglutição, mesmo que esse sintoma possa ser temporário, em alguns casos, e que a dieta por via oral proceda normalmente, não se pode esquecer que essa alteração pode levar a quadros de desnutrição, desidratação e deficiências nutricionais (Neumann \& Adami, 2018).

Quanto aos aspectos alimentares, 76,3\% alimentam-se por via oral em todas as consistências alimentares, 7,9\% por via oral em consistência pastosa e líquida espessado, seguida por vias alternativas de alimentação: 5,3\% por sonda nasoenteral (SNE), 5,3\% por sonda nasogástrica (SNG) e 2,6\% por gastrostomia (GTT).

Quanto aos aspectos de deglutição, 89,5\% apresentam dificuldade para deglutir e 10,5\% não deglutem. A dificuldade para deglutir apresentada pela maioria dos idosos pode estar associada ao tipo do AVC e à extensão da área que foi lesionada, e somam-se a esse quadro, as alterações fisiológicas nas estruturas envolvidas no processo de deglutição provenientes do envelhecimento natural.

Com base nessas etapas, elaborou-se o fluxograma considerando. O fluxograma está apresentado na Figura 1. 
Research, Society and Development, v. 10, n. 8, e20110816978, 2021

(CC BY 4.0) | ISSN 2525-3409 | DOI: http://dx.doi.org/10.33448/rsd-v10i8.16978

Figura 1. Dados da pesquisa realizada pelos autores.

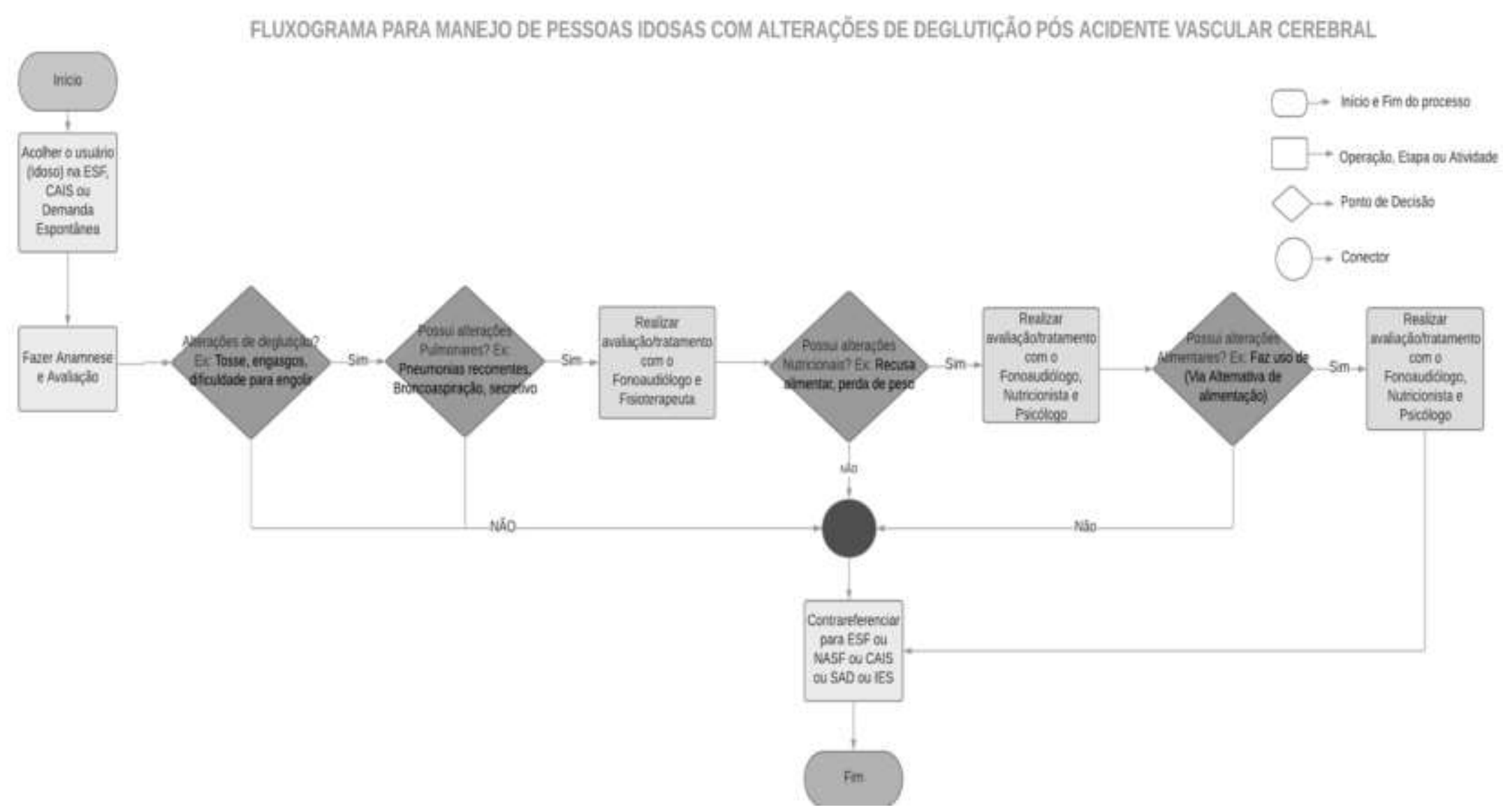

Fonte: Autores. 
O fluxo inicia com o acolhimento da pessoa idosa pós AVC realizado na Estratégia Saúde da Família, Centro de Assistência Integral em Saúde ou a partir de uma demanda espontânea em outro serviço de saúde da rede. Nesses serviços, esse idoso será submetido à anamnese e à avaliação por um profissional de saúde, que identificará as sequelas relacionadas ao AVC e, mais especificamente, observará alterações na deglutição. Não havendo alterações na deglutição, esse idoso deverá ser encaminhado para a Estratégia Saúde da Família, Núcleo Ampliado de Atenção a Saúde da Família, Serviço de Atenção Domiciliar, Centro de Assistência Integral em Saúde e/ou Instituição de Ensino Superior.

Havendo alterações na deglutição, no que se refere a alterações pulmonares, o idoso deverá ser encaminhado ao profissional de fisioterapia e fonoaudiologia para avaliação e tratamento. Encontrando-se alterações nutricionais, o idoso deverá ser encaminhado ao profissional de fonoaudiologia, nutrição e psicologia. Havendo alterações alimentares, o idoso deverá ser encaminhado ao profissional de fonoaudiologia, psicologia e nutrição.

\section{Discussão}

As desordens de deglutição em idosos acometidos por AVC são essencialmente orofaríngeas, relacionadas aos aspectos de tonicidade, mobilidade e sensibilidade das estruturas que estão inadequadas, e, consequentemente, ocorre atenuação dos reflexos de proteção de via aérea, podendo ocorrer tosse e engasgos durante a deglutição como sinais e sintomas. Assim, as dificuldades no ato de engolir encontram-se instaladas na vida alimentar da pessoa idosa, indicando um aumento do risco de pneumonias em virtude de aspiração e ainda a necessidade de via alternativa de alimentação (Silva, 2006).

O fluxograma consiste numa ferramenta que disponibiliza o cuidado ao idoso, a sistematização de terapêuticas no sistema de saúde em uso, em prol da continuidade e qualidade da assistência e da continuidade do cuidado. Faz-se necessário e relevante o desenvolvimento da produção de indicadores deste processo e os resultados, de forma a evidenciar os ganhos em saúde sensíveis aos cuidados que se deseja informar (Celeste \& Alves, 2019).

Com o fluxograma, é possível sistematizar as terapêuticas utilizadas no cuidado à pessoa idosa, tendo em vista a preparação para os encaminhamentos a serem realizados, a reabilitação, a continuidade e qualidade dos cuidados. É de salientar a unanimidade encontrada na definição das especificações que concretizam cada uma das intervenções com integridade referencial para a gestão dos processos de cuidado em saúde, que indica as referidas tomadas de decisão a partir de determinado contexto (Amaya \& Silva, 2016).

O fluxograma como ferramenta no processo de cuidado em saúde ao idoso permite a possibilidade de desenvolver roteiros clínicos que orientam a prática assistencial no sistema de informação em uso. Com este roteiro, é possível, em qualquer momento do cuidado, proporcionar as capacidades e competências adquiridas pelo idoso e pela equipe multidisciplinar, permitindo a qualquer ator do cuidado planificar a intervenção seguinte, sem dar espaço a cuidados padronizados e que não respondam às reais necessidades dos doentes, tendo em vista a reabilitação de forma célere e eficaz (Veras \& Cordeiro, 2013).

O direcionamento clínico é orientado pelas necessidades dos idosos de forma a proporcionar-lhes qualidade no cuidado para a sua reabilitação ser um sucesso. Portanto, os objetivos traçados para o cuidado são realizáveis, o que resultará numa maior visibilidade dos resultados sensíveis aos cuidados de uma equipe multidisciplinar. É considerado um instrumento usado frequentemente com sucesso em sua atuação e com desempenho seguro no processo de cuidado ${ }^{1}$.

A implantação do fluxograma na prática pode ser considerada um benefício, uma vez que ações mais efetivas, equipes capacitadas, tecnologias eficazes e que garantam segurança e celeridade ao serviço prestado ao paciente. Esse instrumento viabiliza o direcionamento de forma assertiva e célere da equipe multidisciplinar no manejo ou direcionamento da pessoa idosa 
que apresenta alterações de deglutição pós Acidente vascular cerebral, priorizando uma reabilitação eficaz e proporcionando qualidade de vida alimentar à pessoa idosa (Aquino \& Vasconcelos, 2017).

A partir dos achados encontrados no estudo de revisão integrativa e na pesquisa documental, foram elencadas as principais alterações na deglutição que foram inseridas no fluxograma: alterações pulmonares, nutricionais e alimentares. Em seguida, foi elaborado o fluxograma para o manejo da pessoa idosa com alterações na deglutição pós-AVC com auxílio do programa informático Lucidchart.

O fluxo inicia com o acolhimento da pessoa idosa pós-AVC realizado na ESF, CAIS ou a partir de uma demanda espontânea em outro serviço de saúde da rede. Nesses serviços, esse idoso será submetido à anamnese e à avaliação por um profissional de saúde, que identificará as sequelas relacionadas ao AVC e, mais especificamente, observará alterações na deglutição. Não havendo alterações na deglutição, esse idoso deverá ser encaminhado para a ESF, NASF, SAD, CAIS e/ou IES.

Havendo alterações na deglutição, no que se refere a alterações pulmonares, o idoso deverá ser encaminhado ao profissional de fisioterapia e fonoaudiologia para avaliação e tratamento. Encontrando-se alterações nutricionais, o idoso deverá ser encaminhado ao profissional de fonoaudiologia, nutrição e psicologia. Havendo alterações alimentares, o idoso deverá ser encaminhado ao profissional de fonoaudiologia, psicologia e nutrição.

\section{Conclusão}

A partir dos objetivos propostos neste estudo, foi possível identificar a prevalência das alterações de deglutição pós AVC em pessoas idosas assistidas em um serviço de atendimento domiciliar, a partir de dados coletados em prontuários, e por conseguinte, a elaboração de um fluxograma para o manejo de pessoas idosas com alterações de deglutição que sofreram Acidente vascular cerebral.

A partir dos dados alcançados com a pesquisa, observou-se que existem várias alterações na deglutição de pessoas idosas pós-AVC que comprometem a saúde e a qualidade de vida dos indivíduos acometidos. E para cada tipo de alteração, é possível uma forma de encaminhamento e acompanhamento para profissionais de saúde diversos que integram a rede de atenção à saúde. Através da atuação de uma equipe multiprofissional, é possível garantir a integralidade do cuidado, promovendo a reabilitação desse idoso.

O fluxograma apresentado neste estudo pode auxiliar os profissionais de saúde que assistem esta demanda, proporcionando um melhor direcionamento, o mais precoce possível, ao profissional fonoaudiólogo, bem como à equipe multidisciplinar, minimizando os danos e complicações e, consequentemente, uma melhor qualidade de vida alimentar dos idosos acometidos por AVC.

Esse estudo também aponta para a importância do devido preenchimento das informações nos prontuários, o que auxilia na construção de novos conhecimentos e, consequentemente, melhorias para os serviços de saúde.

Em relação à aplicabilidade do produto tecnológico, sabe-se que é necessária a realização de investimento em matriciamentos na rede de atenção à saúde, a fim de que todos aqueles que fazem parte deste processo de assistência à saúde tomem o devido conhecimento deste produto, bem como de sua aplicabilidade, e o utilizem em sua prática como instrumento facilitador no cuidado à pessoa idosa.

Como sugestão de novas pesquisas científicas, sugere-se que este fluxograma aqui produzido, seja aplicado pelas diversas categorias profissionais de saúde, para o manejo de pessoas idosas com alterações de deglutição pós-acidente vascular cerebral e que os resultados sejam apresentados a fim de confirmar a aplicabilidade do instrumento. Faz-se necessário que o 
instrumento seja utilizado especialmente por profissionais de saúde, a fim de que, se tenha um olhar holístico sobre a saúde da pessoa idosa, e no seu cuidado dentro da Rede de Atenção à Saúde.

\section{Referências}

Amaya, M. (2016). Construção e validação de conteúdo de checklist para a segurança do paciente em emergência. Rev. Gaúcha Enfermagem, 37(1),687-778. http://www.scielo.br/pdf/rgenf/v37nspe/0102-6933-rgenf-1983-14472016esp2016-68778/

Celeste, L. (2017). Mapeamento da Fonoaudiologia Educacional no Brasil: formação, trabalho e experiência profissional. Rev.CoDAS, 29(1),1-12. http://www.scielo.br/scielo.php?script=sci_arttext\&pid=S231717822017000100309\&lng=e/

Cunha, D. (2016). Contribuição fonoaudiológica em idosos acometidos de acidente vascular Encefálico. Rev. bras. geriatr. gerontol, 10(3), 25-28. https://pesquisa.bvsalud.org/portal/resource/pt/biblio-905351/

Chaves, S.(2019). Disfagia em idosos pós AVC: checklist para rastreamento. Rev. Riase, 5(2),377-390. http://www.revistas.uevora.pt/index.php/saude_envelhecimento/article/view/

Estrela, C. (2018). Metodologia Científica: Ciência, Ensino, Pesquisa. Editora Artes Médicas.

Ludke, M. (2013). Pesquisas em educação: uma abordagem qualitativa. Editora Artes Médicas

Magalhães, L.(2014) Avaliação de linguagem e de deglutição de pacientes hospitalizados após acidente vascular cerebral. Rev. Distúrbios da Comunicação, 16(1), 65-81. https://revistas.pucsp.br/dic/article/view/11621/

Maria, A.(2017). Implantação de fluxograma de atendimento em um Centro de Atenção Psicossocial. Rev. Bras. em Promoção da Saúde, 30(2), 288-293.São Paulo https://periodicos.unifor.br/RBPS/article/view/5819/

Mourão, A. (2016). Frequência e fatores associados à disfagia após acidente vascular cerebral. Rev. Codas, 28(1), 56-64. https://www.scielo.br/scielo.php?pid=S231717822016000100066\&script=sci_arttext./

Mota, L.(2014). Sistemas de informação de enfermagem: Exploração da informação partilhada com os médicos. Rev. Enf. Ref., 4(1), 85-91. http://www.scielo.mec.pt/scielo.php?script=sci_abstract\&pid=S0874-02832014000100010\&lng=pt\&nrm=iso/

Neumann, L. (2016). Sensibilidade gustativa de adultos e idosos.Rev bras geriatr. gerontol. 9(5),797-808. http://www.scielo.br/pdf/rbgg/v19n5/pt_1809-9823rbgg-19-05-00797/

Organização Mundial da Saúde. (2015). Relatório mundial de envelhecimento e saúde. 2 de agosto, 2018. http://sbgg.org.br/wp-content/uploads/

Plassa, B.(2018). Fluxograma descritor no atendimento à pessoa idosa vítima de violência: uma perspectiva interdisciplinar. Revista Esc Anna Nery, 22(4), 221-236. https://www.scielo.br/j/ean/a/hnpYQRy7xzy6MtLWKS8FmWh/

Ribeiro, P. (2015). Relationship between dysphagia, national institutes of health stroke scale score, and predictors of pneumonia after ischemic stroke. $J$. Stroke Cerebrovasc,24(9),2088-2094. https://www.ncbi.nlm.nih.gov/pubmed/26187787. 10.1016/j.jstrokecerebrovasdis.2015.05.009.21/

Silva, L.(2016). Disfagia orofaríngea pós-acidente vascular encefálico no idoso. Rev. bras. geriatr. gerontol. 9(2),93-106. http://www.scielo.br/scielo.php?script=sci_arttext\&pid=S1809-98232006000200093\&lng=pt\&nrm=iso\&tlng=pt.https://dx.doi.org/10.1590/18099823.2006.09028/

Schettino, M.(2020). Desidratação, acidente vascular cerebral e disfagia:revisão sistemática da literatura. Rev.Audiol.Commun, 24(1), 221-235. https://www.scielo.br/scielo.php?pid=S231764312019000100508\&script=sci_arttext/

Sporns, P. (2017).Atrophy of swallowing muscles is associated with severity of dysphagia and age in patients with acute stroke. J. Am. Med. Dir. Assoc., 18(7),635-647. https://www.ncbi.nlm.nih.gov/pubmed/28363443/

Sousa, I.(2018). Reabilitação fonoaudiológica da disfagia orofaríngea após acidente vascular encefálico: revisão de literatura. Journal of Specialist. 1(1), 1-11. http://journalofspecialist.com/jos/index.php/jos/article/view/65/29/

Veras, R. (2013). Modelos de atenção à saúde do idoso: repensando o sentido da prevenção. Physis Revista de Saúde Coletiva, 23(4), 1189-1213. https://www.scielo.br/j/physis/a/PNKYtHHScFWKVHpvnk7XmRf/?lang=pt/

Winstein, C.(2016). Guidelines for adult stroke rehabilitation and recovery: a guideline for healthcare professionals from the American Heart Association.American Stroke Association, 47(1), 1224-1236. https://www.ahajournals.org/doi/full/10.1161/STR.0000000000000098/ 